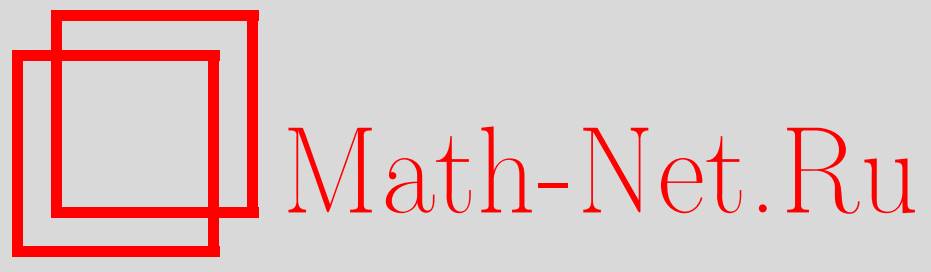

А. В. Кочергин, Невырожденные седла и отсутствие перемешивания II, Матем. заметки, 2007, том 81, выпуск $1,145-148$

DOI: https://doi.org/10.4213/mzm3527

Использование Общероссийского математического портала Math-Net.Ru подразумевает, что вы прочитали и согласны с пользовательским соглашением http://www . mathnet.ru/rus/agreement

Параметры загрузки:

IP : 3.93 .64 .190

26 апреля 2023 г., 12:34:31

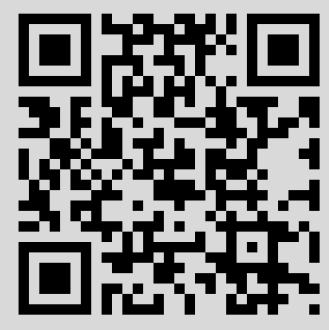




\section{Невырожденные седла и отсутствие перемешивания II}

\section{А. В. Кочергин}

1. Такие специальные потоки возникают при рассмотрении гладких потоков на компактных поверхностях, эргодических относительно меры с гладкой плотностью и имеющих неподвижные точки. Как показано в [1], время прохождения траектории через окрестность невырожденной седловой точки гладкого потока имеет порядок логарифма минимального расстояния от этой точки, поэтому в случае, когда поток имеет конечное число неподвижных точек и все они суть невырожденные седла, поток представляется в виде специального потока, построенного, вообще говоря, по перекладыванию отрезков окружности $\mathbb{S}^{1}=\mathbb{R} / \mathbb{Z}$ и функции вида

$$
f(x)=f_{0}(x)+\sum_{i=1}^{N}\left(-A_{i} \ln \left\{x-\bar{x}_{i}\right\}-B_{i} \ln \left\{\bar{x}_{i}-x\right\}\right), \quad A_{i}, B_{i}>0,
$$

где $f_{0}$ - функция ограниченной вариации и $\sum_{i=1}^{N} A_{i}=\sum_{i=1}^{N} B_{i}$. В отдельных случаях рассматриваемое перекладывание является поворотом окружности [2].

Функцию вида (1) с ограничением $\sum_{i=1}^{N} A_{i}=\sum_{i=1}^{N} B_{i}$ мы называем симметричной функцией с логарифмическими особенностями.

Теорема 1. Специалъный поток, построенный по любому повороту окружности и симметричной функции с логарифмическими особенностями, не перемешивает.

Аналогичный результат, но для углов, допускающих аппроксимацию рациональными дробями со скоростью const $/\left(q^{2} \ln q\right)$, получен в работе автора [1]. М. Леманчик [3] заметил одну очень простую ситуацию, когда утверждение верно для любого иррационального угла - это случай симметричных особенностей, когда $A_{i}=B_{i}$ для любого $i$.

Заметим, что если все особенности функции степенные, что соответствует вырожденным неподвижным точкам в потоке на поверхности, то специальный поток над эргодическим перекладыванием отрезков перемешивает [4].

Для гладких асимметричных функций с логарифмическими особенностями, когда $\sum_{i=1}^{N} A_{i} \neq \sum_{i=1}^{N} B_{i}$ (такие потоки возникли в работе В. И. Арнольда [5]), напротив, доказано наличие перемешивания как для некоторого класса диофантовых чисел вращения, т.е. достаточно плохо аппроксимируемых рациональными числами [6], [7], так и для достаточно хорошо аппроксимируемых, лиувиллевых, чисел при некоторых ограничениях на коэффициенты $A_{i}$ и $B_{i}[8]$.

Остается открытым вопрос о существовании неперемешивающего специального потока, построенного по какому-нибудь эргодическому повороту окружности и асимметричной функции с логарифмическими особенностями. Ничего не известно также о перемешивающих свойствах специального потока над перекладыванием отрезков с "крышей", имеющей логарифмические особенности.

2. Через $T$ мы будем обозначать поворот окружности $\mathbb{S}^{1}=\mathbb{R} / \mathbb{Z}$ на угол $\rho, \rho=$ $\left[k_{1}, \ldots, k_{n}, \ldots\right]$ - разложение $\rho$ в цепную дробь, $p_{n} / q_{n}-n$-ю подходящую к $\rho$ дробь, $\delta_{n}=q_{n} \rho-p_{n}$. Известно, что $|\delta|<1 / q_{n+1}$ (см. [9]).

Работа выполнена при частичной поддержке программы "Ведущие научные школы РФ", грант № 6849.2006.1.

(C) А.В. Кочергин, 2007 
Основным инструментом изучения перемешивающих свойств специального потока над поворотом окружности служат “биркгофовы суммы”, построенные по $T$ и функции $f$ : для $r \in \mathbb{N}$

$$
f^{r}(x)=f_{T}^{r}(x)=\sum_{k=0}^{r-1} f\left(T^{k} x\right), \quad f^{-r}(x)=-f^{r}\left(T^{-r} x\right), \quad f^{0}(x) \equiv 0 .
$$

Через $O_{f, n}(\eta)$ обозначим объединение $\left(\eta / q_{n}\right)$-окрестностей особых точек биркгофовой суммы $f^{q_{n}}$. Заметим, что $\mu\left(O_{f, n}(\eta)\right) \leqslant 2 N \eta$, где $N$ - число особых точек функции $f$.

Доказательство отсутствия перемешивания сводится к следующей теореме о поведении биркгофовых сумм симметричной функции $f$ с логарифмическими особенностями.

Теорема 2. Для любого $\eta>0$ существует такое число $M>0$, что для бесконечной подпоследовательности номеров $n$ значения каждой биркгофовой суммы $f^{q_{n}}$ на множестве $\mathbb{S}^{1} \backslash O_{f, n}(\eta)$ содержатся в объединении не более, чем $5^{2 N-1}$ отрезков, каждый из которых не длиннее $M$.

ДокАЗАТЕЛЬСтво. Симметричную функцию $f$ с $N$ особыми точками можно представить в виде суммы $f=f_{0}+\sum_{i=1}^{2 N-1} c_{i} g_{i}$ с неотрицательными коэффициентами $c_{i}$, где каждая из функций $g_{i}$ является сдвигом функции $\mathbb{S}^{1} \rightarrow \mathbb{R}$ вида $g(x)=-\ln \{x-a\}-$ $\ln \{-a-x\}$ для некоторого $a$. Нетрудно видеть, что теорему 2 достаточно доказать для функции $g$.

Укажем правило выбора подпоследовательности номеров подходящих дробей, для которых будет показана справедливость теоремы. Если последовательность неполных частных разложения $\rho$ в цепную дробь ограничена, то выберем подпоследовательность номеров $n$, для которых $q_{n}|\delta|<1 / 2$ (такой номер есть среди каждых двух последовательных номеров [9]). Если же последовательность неполных частных $k_{n}$ не ограничена, то выберем подпоследовательность номеров $n$, для каждого из которых $k_{n+1} \geqslant \max \left(k_{1}, \ldots, k_{n}\right)$. В этом случае $q_{n}|\delta|<q_{n} / q_{n+1}<1 / k_{n+1}<1 / 2$, начиная с некоторого номера.

Фиксируем $n$. Обозначим $K_{n}=\max \left(k_{1}, \ldots, k_{n}\right)$. В дальнейшем для краткости индекс $n$ будем опускать: будем писать просто $p / q, \delta=q \rho-p, K$, а вместо $O_{g, n}(\eta)$ будем писать $O(\eta)$.

Для удобства обозначим $u(x)=-\ln x, g(x)=u(\{x-a\})+u(\{-x-a\})$. Тогда

$$
g^{q}(x)=\sum_{r=0}^{q-1} u(\{x+r \rho-a\})+\sum_{r=0}^{q-1} u(\{-(x+r \rho)-a\}) .
$$

Положим

$$
\begin{aligned}
& a=\frac{A}{q}+\frac{a_{0}}{q}, \quad A \in \mathbb{Z}_{+}, \quad 0 \leqslant A \leqslant q-1, \quad-\frac{1}{2}<a_{0} \leqslant \frac{1}{2}, \\
& x=\frac{X}{q}+\frac{t}{q}, \quad X \in \mathbb{Z}_{+}, \quad 0 \leqslant X \leqslant q-1, \quad-\frac{1}{2}<t \leqslant \frac{1}{2} .
\end{aligned}
$$

Кроме того, перенумеруем слагаемые в (2) следующим образом. В первой сумме положим

$$
s=\{X-A+r p\}_{q} \Longleftrightarrow r=\pi_{X}^{+}(s)=\left\{p^{-1}(-X+A+s)\right\}_{q},
$$

где $p^{-1}$ вычет, обратный к $p(\bmod q),\{\cdot\}_{q}=q\{\cdot / q\}$. Аналогично, для второй суммы положим

$$
s=\{-X-A-r p\}_{q}, \quad \pi_{X}^{-}(s)=\left\{p^{-1}(-X-A-s)\right\}_{q} .
$$

В итоге для $x \in((X-1 / 2) / q,(X+1 / 2) / q]$ получим $g^{q}(x)=\varphi_{X, 1}(t)+\varphi_{X, 2}(t)$, где

$$
\begin{aligned}
\varphi_{X, 1}(t) & =\sum_{s=2}^{q-2}\left(u\left(\frac{1}{q}\left(t-a_{0}+\pi_{X}^{+}(s) \delta+s\right)\right)+u\left(\frac{1}{q}\left(-t-a_{0}-\pi_{X}^{-}(s) \delta+s\right)\right)\right), \\
\varphi_{X, 2}(t) & =\sum_{s=0,1, q-1}\left(u\left(\frac{\left\{t-a_{0}+\pi_{X}^{+}(s) \delta+s\right\}_{q}}{q}\right)+u\left(\frac{\left\{-t-a_{0}-\pi_{X}^{-}(s) \delta+s\right\}_{q}}{q}\right)\right) .
\end{aligned}
$$


Для каждого $X$ обозначим через $O_{X}^{\prime}(\eta)$ объединение $\eta$-окрестностей особых точек функции $\varphi_{X, 2}$. В силу (3) условие $x \in O(\eta) \cap(X / q-1 /(2 q), X / q+1 /(2 q)]$ равносильно $t \in O_{X}^{\prime}(\eta)$.

Справедливость теоремы 2 для функции $g$ легко следует из следующих двух лемм.

Лемма 1. Для любого $\eta \in(0,1 / 2)$ существуют не более пяти отрезков $I_{n, 1}, \ldots, I_{n, 5}$, длина каждого из которых не более $5 \ln (1 / \eta)+6$ и объединение которых содержит множества $\varphi_{X, 2}\left((-1,1] \backslash O_{X}^{\prime}(\eta)\right)$ для всех $X=0, \ldots, q-1$.

Доказательство леммы основано на том, что функция $\varphi_{X, 2}$ может иметь в интервале $(-1 / 2,1 / 2)$ не менее одной и не более пяти особых точек.

Лемма 2. Существует отрезок $I_{n, 0}$ длины не более, чем $40 K / k_{n+1}+11$, содержащий множества значений функиий $\varphi_{X, 1}$ для всех $X=0, \ldots, q-1$.

Справедливость леммы 1 очевидно следует из следующих двух оценок.

Лемма 3. Для любого $X=0, \ldots, q-1$ и любого $t \in(-1 / 2,1 / 2)$ выполнено неравенство $\left|\varphi_{X, 1}^{\prime}(t)\right|<7$.

Аналогичная лемма доказана, например, в [7].

ЛЕмма 4. Множество $\bigcup_{X=0}^{q-1} \varphi_{X, 1}(0)$ содержится в отрезке длины не более, чем $40 K / k_{n+1}+4$.

ДокАзАтЕльство. Преобразуем (4). Воспользовавшись разложением логарифма и учитывая равенство

$$
\begin{aligned}
\pi_{X}^{+}(s)-\pi_{X}^{-}(s) & =\left\{p^{-1}(2 A+2 s)\right\}_{q}-\frac{q}{2}+\frac{q}{2} \varepsilon_{X}(s), \\
\text { где } \quad \varepsilon_{X}(s)=1 \quad \text { при } \pi_{X}^{+}(s) & \geqslant \pi_{X}^{-}(s), \quad \varepsilon_{X}(s)=-1 \quad \text { при } \pi_{X}^{+}(s)<\pi_{X}^{-}(s),
\end{aligned}
$$

можно показать, что

$$
\left|\varphi_{X, 1}(0)-\Phi\right| \leqslant \frac{q \delta}{2} \Sigma+2
$$

где

$$
\Phi=\sum_{s=2}^{q-2}\left(2 \ln \frac{q}{-a_{0}+s}+\frac{\left(\left\{p^{-1}(2 A+2 s)\right\}_{q}-q / 2\right) \delta}{-a_{0}+s}\right)
$$

- константа, не зависящая от $X$ и $t$ (и, тем самым, от $x$ ), и $\Sigma=\sum_{s=2}^{q-2} \varepsilon_{X}(s) / s$.

Применим к $\Sigma$ преобразование Абеля: положим $\sigma(s)=\sum_{r=2}^{s} \varepsilon_{X}(s)$; тогда

$$
\Sigma=\frac{\sigma(q-2)}{q-2}+\sum_{s=2}^{q-3} \frac{\sigma(s)}{s(s+1)} .
$$

Осталось доказать следующую лемму.

Лемма 5. Для любого $s=3, \ldots, q-2$

$$
|\sigma(s)| \leqslant 8 K(3 \ln s+2) .
$$

ДоказАтельство. Положим $\alpha=\left\{p^{-1} X / q\right\}, \beta_{0}=\left\{p^{-1} A / q\right\}, \beta=\left\{p^{-1} / q\right\}, \beta_{s}=\left\{\beta_{0}+\right.$ $s \beta\}$. Тогда условие $\pi_{X}^{+}(s) \geqslant \pi_{X}^{-}(s)$ равносильно условию $\beta_{s} \in F$, где $F=[0, \alpha) \cup[1 / 2,1-\alpha]$ при $0 \leqslant \alpha \leqslant 1 / 2$, или $F=[0,1-\alpha] \cup[1 / 2, \alpha)$ при $1 / 2<\alpha<1$. Множество $F$ зависит от $X$, но мы получим оценку, от $X$ не зависящую. Положим $\widetilde{F}(z)=1$ при $z \in F$, $\widetilde{F}(z)=-1$ при $z \notin F$. Очевидно, что $\mu(F)=1 / 2, \int_{\mathbb{S}^{1}} \widetilde{F}(z) d z=0, \operatorname{Var}(\widetilde{F}) \leqslant 8$. В этих обозначениях $\sigma(s)$ можно интерпретировать как биркгофову сумму, определенную по функции $\widetilde{F}$ и повороту $\widetilde{T}$ окружности на угол $\beta: \sigma(s)=\widetilde{F}^{s-1}\left(\beta_{0}+2 \beta\right)$. В силу 
соотношения $q_{n} p_{n-1}-p_{n} q_{n-1}=(-1)^{n}$ (см. [9]) имеем $\beta=q_{n-1} / q_{n}$ при нечетном $n$ и $\beta=1-q_{n-1} / q_{n}$ при четном $n$. Рассмотрим случай $\beta=q_{n-1} / q_{n} ;$ другой случай сводится к этому. Согласно [9] $q_{n-1} / q_{n}=\left[k_{n}, \ldots, k_{1}\right]$. Значит, все неполные частные разложения $\beta$ не превосходят $K$.

Неравенство (5) теперь следует из неравенства Данжуа

$$
\left|\widetilde{F}_{\widetilde{T}}^{q_{i}^{\prime}}\left(z_{1}\right)-\widetilde{F}_{\widetilde{T}}^{q_{i}^{\prime}}\left(z_{2}\right)\right| \leqslant \operatorname{Var}(\widetilde{F}) \leqslant 8
$$

и разложения Островского числа $s-1$ по знаменателям $q_{i}^{\prime}$ подходящих к $q_{n-1} / q_{n}$ дробей. Лемма 5 , а вместе с ней и теорема 2 , доказаны.

Подробное доказательство предполагается опубликовать в Трудах Математического института им. В. А. Стеклова.

\section{СПИСОК ЦИТИРОВАННОЙ ЛИТЕРАТУРЫ}

[1] А. В. Кочергин, Матем. заметки, 19:3 (1976), 453-468. [2] А. А. Блохин, Тр. ММО, 27 (1972), 113-128. [3] M. Lemańczyk, Colloq. Math., 84/85 (2000), 29-41. [4] A. В. Koчергин, Матем. сб., 96 (138):3 (1975), 471-502. [5] В.И. Арнольд, Функцион. анализ и его прилож., 25:2 (1991), 1-12. [6] Я.Г. Синай, К. М. Ханин, Функиион. анализ и его прилож., 26:3 (1992), 1-21. [7] А. В. Кочергин, Матем. сб., 194:8 (2003), 83-112. [8] А. В. Кочергин, Матем. сб., 195:9 (2004), 19-36. [9] А. Я. Хинчин, Цепные дроби, Физматгиз, М., 1961.

\section{А. В. Кочергин}

Поступило

Московский государственный университет

21.07.2006

им. М. В. Ломоносова 